\title{
Electron acceleration and radio emission following the early interaction of two coronal mass ejections ${ }^{\star}$
}

\author{
D. E. Morosan ${ }^{1}$, E. Palmerio ${ }^{1,2}$, J. E. Räsänen ${ }^{1}$, E. K. J. Kilpua ${ }^{1}$, J. Magdalenić ${ }^{3}$, B. J. Lynch $^{2}$, A. Kumari ${ }^{1}$, \\ J. Pomoell ${ }^{1}$, and M. Palmroth ${ }^{1,4}$ \\ ${ }^{1}$ Department of Physics, University of Helsinki, PO Box 64, 00014 Helsinki, Finland \\ e-mail: diana.morosan@helsinki.fi \\ 2 Space Sciences Laboratory, University of California-Berkeley, Berkeley, CA 94720, USA \\ 3 Solar-Terrestrial Centre of Excellence-SIDC, Royal Observatory of Belgium, 1180 Brussels, Belgium \\ ${ }^{4}$ Space and Earth Observation Centre, Finnish Meteorological Institute, PO Box 503, 00014 Helsinki, Finland
}

Received 1 July 2020 / Accepted 19 August 2020

\begin{abstract}
Context. Coronal mass ejections (CMEs) are large eruptions of magnetised plasma from the Sun that are often accompanied by solar radio bursts produced by accelerated electrons.

Aims. A powerful source for accelerating electron beams are CME-driven shocks, however, there are other mechanisms capable of accelerating electrons during a CME eruption. So far, studies have relied on the traditional classification of solar radio bursts into five groups (Type I-V) based mainly on their shapes and characteristics in dynamic spectra. Here, we aim to determine the origin of moving radio bursts associated with a CME that do not fit into the present classification of the solar radio emission.

Methods. By using radio imaging from the Nançay Radioheliograph, combined with observations from the Solar Dynamics Observatory, Solar and Heliospheric Observatory, and Solar Terrestrial Relations Observatory spacecraft, we investigate the moving radio bursts accompanying two subsequent CMEs on 22 May 2013. We use three-dimensional reconstructions of the two associated CME eruptions to show the possible origin of the observed radio emission.

Results. We identified three moving radio bursts at unusually high altitudes in the corona that are located at the northern CME flank and move outwards synchronously with the CME. The radio bursts correspond to fine-structured emission in dynamic spectra with durations of $\sim 1 \mathrm{~s}$, and they may show forward or reverse frequency drifts. Since the CME expands closely following an earlier CME, a low coronal CME-CME interaction is likely responsible for the observed radio emission.

Conclusions. For the first time, we report the existence of new types of short duration bursts, which are signatures of electron beams accelerated at the CME flank. Two subsequent CMEs originating from the same region and propagating in similar directions provide a complex configuration of the ambient magnetic field and favourable conditions for the creation of collapsing magnetic traps. These traps are formed if a CME-driven wave, such as a shock wave, is likely to intersect surrounding magnetic field lines twice. Electrons will thus be further accelerated at the mirror points created at these intersections and eventually escape to produce bursts of plasma emission with forward and reverse drifts.
\end{abstract}

Key words. Sun: corona - Sun: radio radiation - Sun: particle emission - Sun: coronal mass ejections (CMEs)

\section{Introduction}

One of the most prominent sources of particle acceleration in our Solar System are large eruptions of magnetised plasma from the Sun called coronal mass ejections (CMEs). When they propagate faster than the characteristic speed of the ambient medium, they drive shock waves where electrons can be accelerated to high energies. In addition, the associated magnetic reconfiguration processes during the eruption can lead to the acceleration of particles. The accelerated particles can in turn generate emission at radio wavelengths through the plasma emission mechanism (e.g. Klassen et al. 2002).

Shocks driven by CMEs can be observed in white light as large, extended structures of fainter emission surrounding the brighter CME bubble (Vourlidas et al. 2003, 2013). CME shocks are usually much larger than the underlying magnetic flux rope (e.g. Vourlidas et al. 2013), consisting of a bubble-like structure surrounding the flux rope and CME ejecta (Kwon et al. 2014; Liu et al. 2019). However, other regions are associated with CMEs

\footnotetext{
^ Movies are available at https://www . aanda.org
}

that can accelerate electrons, such as reconnecting current sheets forming at the wake of CMEs (Kahler \& Hundhausen 1992) or magnetic traps that formed due to complex magnetic field configurations following CME eruptions (Magdalenić et al. 2002). Collapsing magnetic traps, in particular, have been previously suggested as a mechanism to further accelerate protons and electrons due to the expansion of the CME shock into the surrounding coronal field lines (Magdalenić et al. 2002; Pomoell et al. 2008; Kong et al. 2012). Strong particle acceleration has also been observed in extreme space weather events as a result of CME-CME interactions at larger heights in the corona (Ding et al. 2013; Gopalswamy et al. 2016), however it is unclear if these types of interactions cause the enhancement of accelerated particles (Richardson et al. 2003). Some of these events are also accompanied by significant radio emission, such as interplanetary radio bursts (Gopalswamy et al. 2016; Liu et al. 2017; Palmerio et al. 2019; Martínez Oliveros et al. 2012).

The most obvious manifestations of shocks at radio wavelengths are a class of radio bursts called Type II bursts, which consist of slowly drifting emission lanes observed in dynamic spectra at the fundamental and harmonic of the plasma frequency 
(Mann et al. 1996; Nelson \& Melrose 1985). Type II emission sources are closely associated with expanding CME-driven shocks in the corona (Stewart et al. 1974; Martínez Oliveros et al. 2012; Liu et al. 2009; Zucca et al. 2018). Type II bursts can often show split-band lanes (Vršnak et al. 2001, 2002) and numerous fine structures composing the emission lanes (Magdalenić et al. 2020). Fine-structured bursts called 'herringbones', which can accompany Type II bursts or occur on their own (Holman \& Pesses 1983; Cairns \& Robinson 1987; Cane \& White 1989), also represent individual electron beams accelerated by a CME shock (Carley et al. 2013; Morosan et al. 2019a). Type IIs and herringbones can be used to study the shock kinematics either through their drift rate observed in dynamic spectra, through radio images, or through multi-point observations, since the emission sources are expected to move in the direction of the CME expansion (e.g. Reiner et al. 2007; Liu et al. 2013; Magdalenić et al. 2014; Morosan et al. 2019a; Zhao et al. 2019; Maguire et al. 2020).

However, there are other radio bursts accompanying CMEs that can also show propagation associated with the CME expansion, namely moving Type IV radio bursts (Boischot 1957). Type IV bursts are a broadband continuum emission, often with superimposed fine structures (e.g. Magdalenić et al. 2006; Bouratzis et al. 2016). These bursts are commonly believed to be generated either by electrons trapped inside the CME flux rope emitting gyro-synchrotron radiation (Dulk 1973; Bastian et al. 2001), electrons accelerated at a reconnecting current sheet forming at the wake of an erupting flux rope, thereby generating bursts of plasma emission (Kahler \& Hundhausen 1992; Vršnak et al. 2003; Morosan et al. 2019b), or emission at the plasma frequency from inside the CME (Stewart et al. 1978; Vasanth et al. 2019). Recent observations have also shown moving Type IV sources that are closely related with the propagation of CME flanks (Morosan et al. 2020a) and Type II-like lanes have also been identified inside a Type IV continuum emission (Chrysaphi et al. 2018). It is therefore feasible that numerous other signatures associated with CMEs that are caused by fast electron beams have been overlooked as a result of adhering to the classical categorisation scheme of solar radio bursts.

The availability of observations from the Solar Terrestrial Relations Observatory (STEREO; Kaiser et al. 2008) in recent times has brought a third dimension to the studies of eruptive events and has allowed us to better understand the relationship between CMEs and associated radio emission (Mancuso et al. 2019; Morosan et al. 2019a, 2020b; Chrysaphi et al. 2020). Significant plane-of-sky projection effects of radio sources have been identified, even in observations of CMEs originating on the solar limb. Therefore, a three-dimensional picture of the radio emission relative to the $\mathrm{CME}$ is necessary to determine the locations of electron acceleration regions. At larger distances, triangulation techniques have so far been used to determine the location of Type II radio sources (e.g. Martínez Oliveros et al. 2012). So far, the 3D location of electron acceleration regions in relation to the propagating $\mathrm{CME}$ structure and ambient corona early during the eruption is poorly known, largely due to limitations of the previously available observational capabilities.

In this paper, we present the first report of moving radio bursts observed at unusually high altitudes $\left(1.5-2.2 R_{\odot}\right.$ at $150 \mathrm{MHz}$ ) and associated with two subsequent CMEs that erupted on 22 May 2013. These bursts do not fit in either the Type II nor Type IV classifications. Using a combination of multi-spacecraft observations and $3 \mathrm{D}$ reconstructions we are able to show that the moving bursts originate at a location where the CME propagates in the wake of an earlier CME, presumably creating conditions where electrons can be accelerated effectively in contracting magnetic bottles. In Sect. 2, we give an overview of the observations and employed data analysis techniques. In Sect. 3, we present the results, which are further discussed in Sect. 4. The conclusions are presented in Sect. 5.

\section{Observations and data analysis}

\subsection{Radio emission}

On 22 May 2013, three moving radio sources were observed in images from the Nançay Radioheliograph (NRH; Kerdraon et al. 1997). The moving radio sources are labelled in order of their appearance in Fig. 1: Source 1 (top panels), Source 2 (middle panels), and Source 3 (bottom panels). These bursts are shown as radio intensity contours at 150 (orange) and $173 \mathrm{MHz}$ (green), overlaid on running-difference images at $211 \AA$ from the Atmospheric Imaging Assembly (AIA; Lemen et al. 2012) onboard the Solar Dynamics Observatory (SDO; Pesnell et al. 2012). Few other stationary radio sources were observed at the same time but at different locations, closer to the active region (see e.g. the other radio contours in Fig. 1 that are not labelled by arrows).

In the combined dynamic spectra from the Radio and Plasma Wave Investigation (SWAVES; Bougeret et al. 2008) onboard STEREO, the Nançay Decametric Array (NDA; Boischot et al. 1980), and the ORFEES (Observation Radio pour FEDOME et l'Étude des Éruptions Solaires) radio spectrograph, an intense radio event was observed over a wide frequency band. The radio emission started at $\sim 13: 00$ UT and lasted for a few hours (Figs. 2a-c).

At frequencies below $100 \mathrm{MHz}$, numerous Type II lanes were observed (Figs. 2a-b), indicating the presence of a shock wave. The kinematics of these Type II lanes in relation to the associated CME (presented in the following section) were studied in Mäkelä et al. (2016) and Palmerio et al. (2019). In the same frequency range, a prominent Type IV burst was observed, lasting for up to 1 hour (Fig. 2b). Drifting structures, possibly other Type II lanes, were superimposed on the Type IV continuum. At frequencies above $140 \mathrm{MHz}$, a short duration ( $\sim 30$-min), broadband continuum emission was observed starting from $\sim 13: 10$ UT (labelled as a Type IV in Fig. 2c). Shortly after the first Type II lanes, a group of Type III bursts was observed in the STEREO/SWAVES frequency range. The preceding radio emission (ORFEES dynamic spectrum in Fig. 2c) is dominated by fine-structured bursts, most of which are Type I bursts constituting a noise storm. The noise storm originated from an unrelated active region located on the eastern solar hemisphere. The majority of the radio emission was observed during the rise time of an M5.0-class flare (the peak of the X-ray flux occurred at 13:32 UT in Fig. 2d from the Geostationary Environmental Satellites; GOES). The most intense Type II lanes were also observed during the rise time of the flare, which indicates the presence of an early shock propagating in the low corona. However, both the Type II and Type IV emissions continue during the decay phase of the flare, with the Type II lanes in particular observed for longer than the low-frequency Type IV burst. All these aspects indicate a long-lasting electron acceleration process as the CME propagates further out into interplanetary space.

\subsection{Eruptive event}

The AIA images with overlaid NRH radio sources (Fig. 1) show that the moving radio bursts occur concurrently with a CME. The 


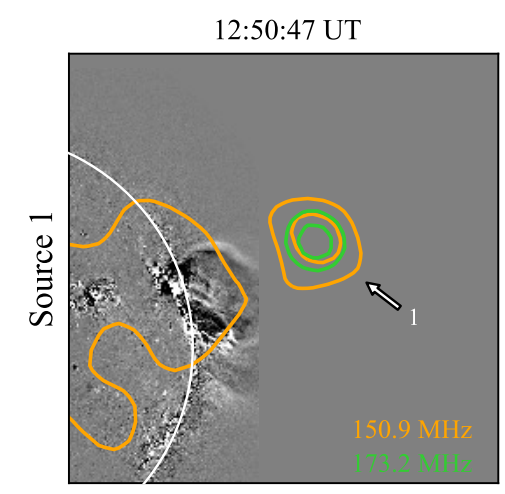

13:01:59 UT

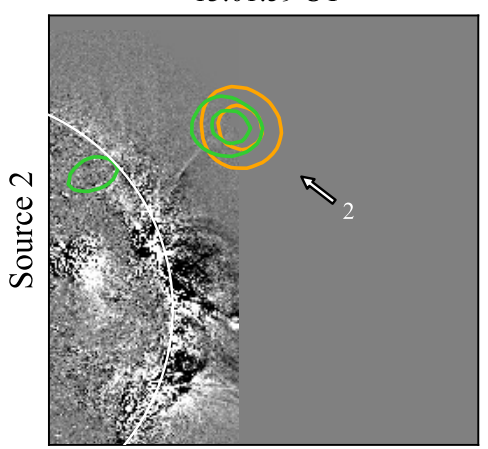

13:17:11 UT

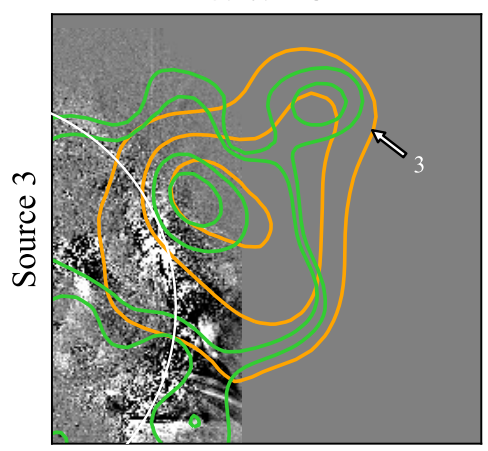

12:53:47 UT

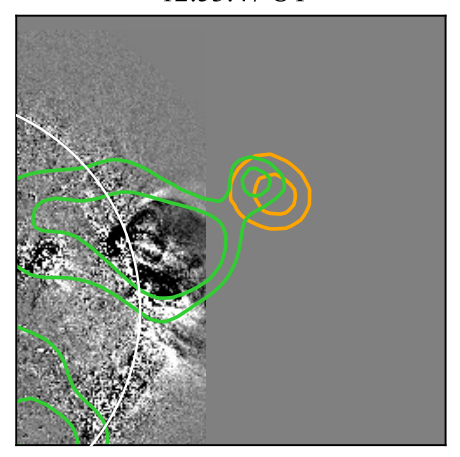

13:06:11 UT

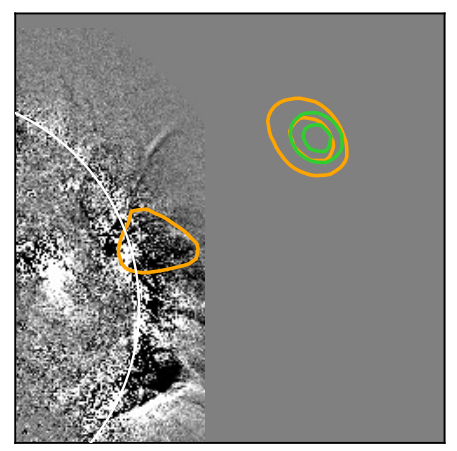

13:19:11 UT

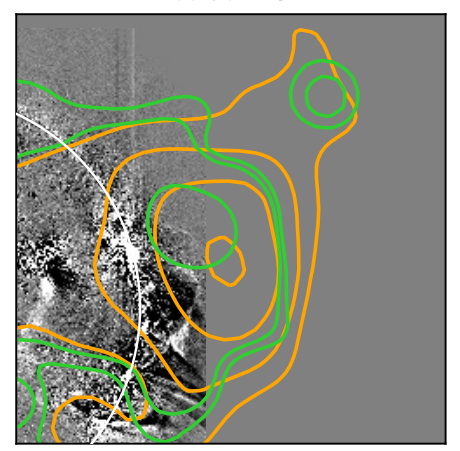

$12: 57: 35$ UT

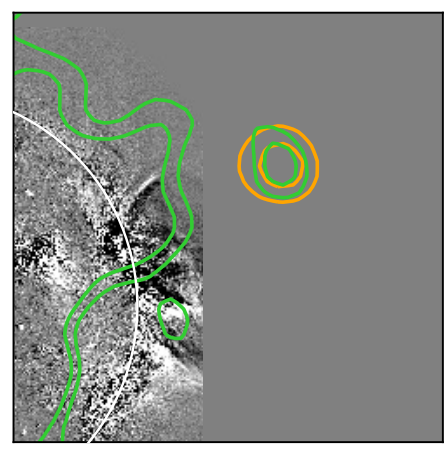

13:07:59 UT

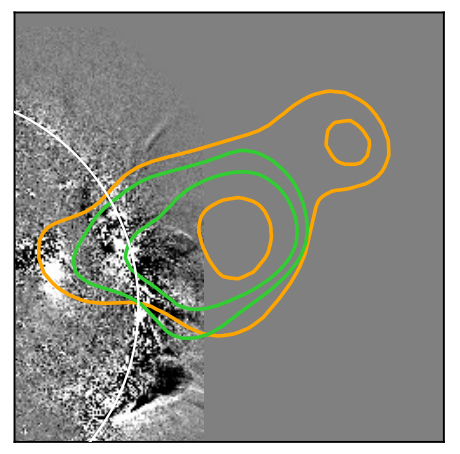

13:20:23 UT

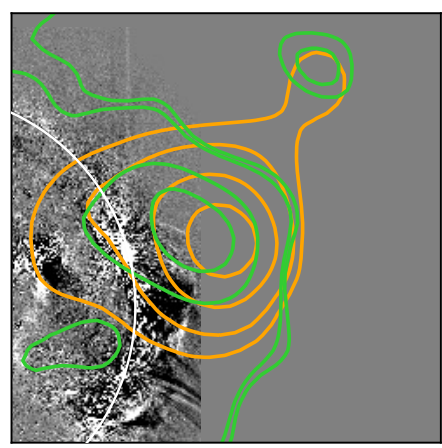

Fig. 1. Moving radio bursts associated with the expansion of the 22 May $2013 \mathrm{CME}$ in the solar corona. NRH radio contours at $150.9 \mathrm{MHz}$ (orange) and 173.2 MHz (green) are overlaid on AIA 211 Å running-difference images in each panel. Three moving radio bursts were identified and labelled as Source 1 (top panels), Source 2 (middle panels), and Source 3 (bottom panels). The AIA images show the evolution of the CME through time.

radio sources appear to follow the propagation direction of the CME which accompanies the GOES M5.0-class flare (Fig. 2d). The NRH moving radio bursts seem to be associated with the northern CME flank (for a full evolution of the moving radio bursts and the CME, see Movie 1 online).

The CME was well observed in remote-sensing data from instruments onboard the Solar and Heliospheric Observatory (SOHO; Domingo et al. 1995), the Project for On Board Autonomy 2 (PROBA2; Santandrea et al. 2013), and SDO that are located near Earth. The CME was also observed by the twin STEREO spacecraft, separated from Earth by $137^{\circ}$ and $141^{\circ}$, respectively. Figure 3 shows the CME as it propagates through the solar corona from the perspectives of Earth (Fig. 3a) and STEREO-A (Fig. 3b). The first observation of the CME in white-light coronagraph images was at 12:55 UT by STEREOA's inner coronagraph COR1, which is part of the Sun Earth Connection Coronal and Heliospheric Investigation (SECCHI; Howard et al. 2008) suite. From Earth's perspective, the CME was observed first at 13:25 UT with The Large Angle Spectrographic Coronagraph (LASCO; Brueckner et al. 1995) C2 instrument onboard SOHO.
Sun-monitoring spacecraft in Earth's orbit, such as SOHO and SDO, were located at ideal locations from STEREO-A such that the CME is well observed from different sides. SOHO and SDO are separated at this time by $137^{\circ}$ from STEREOA, with their mid-point at $68.5^{\circ}$ (see the inset in Fig. 3). The CME longitude deduced from observations is $+67^{\circ}$ (Palmerio et al. 2019) in Stonyhurst coordinates. Therefore, the locations of the spacecraft close to Earth and STEREO-A are almost perfectly symmetrical with respect to the CME propagation direction (Fig. 3). The CME is shown from Earth's perspective in a combined image at $\sim 13: 25$ UT from the Sun-Watcher with Active Pixel System and Image Processing (SWAP; Seaton et al. 2013) onboard PROBA2, LASCO/C2, and LASCO/C3 (Fig. 3a). From the perspective of STEREO-A, the CME is shown in a combined image at $\sim 13: 25$ UT from STEREO-A's Extreme Ultraviolet Imager (EUVI), inner coronagraph COR1, and outer coronagraph COR2 (Fig. 3b).

Careful investigation of images from LASCO's outer coronagraph C3 and STEREO-A's outer coronagraph COR2 (Fig. 3) shows that the CME did not expand through the undisturbed solar corona, but behind an ongoing earlier CME. The full 


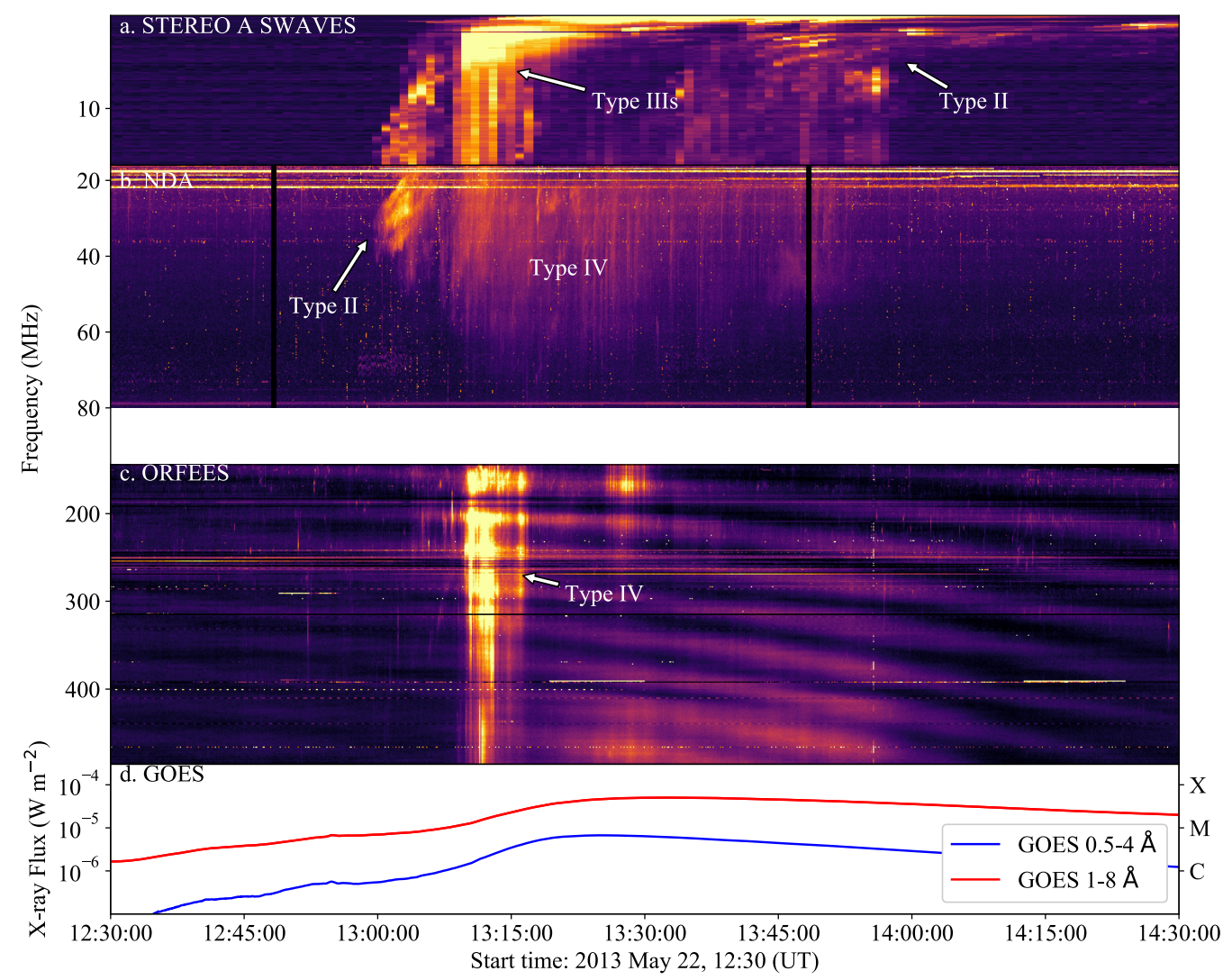

Fig. 2. Dynamic spectrum of the radio bursts associated with the 22 May 2013 CME and solar X-ray flux. (a), (b), (c) Composite dynamic spectrum from SWAVES-A, NDA, and ORFEES showing the radio activity associated with the CME in the following frequency ranges: 1-16, 16-80, and 144-484 MHz, respectively. (d) GOES solar X-ray flux indicating the occurrence of an M5.0 solar flare.

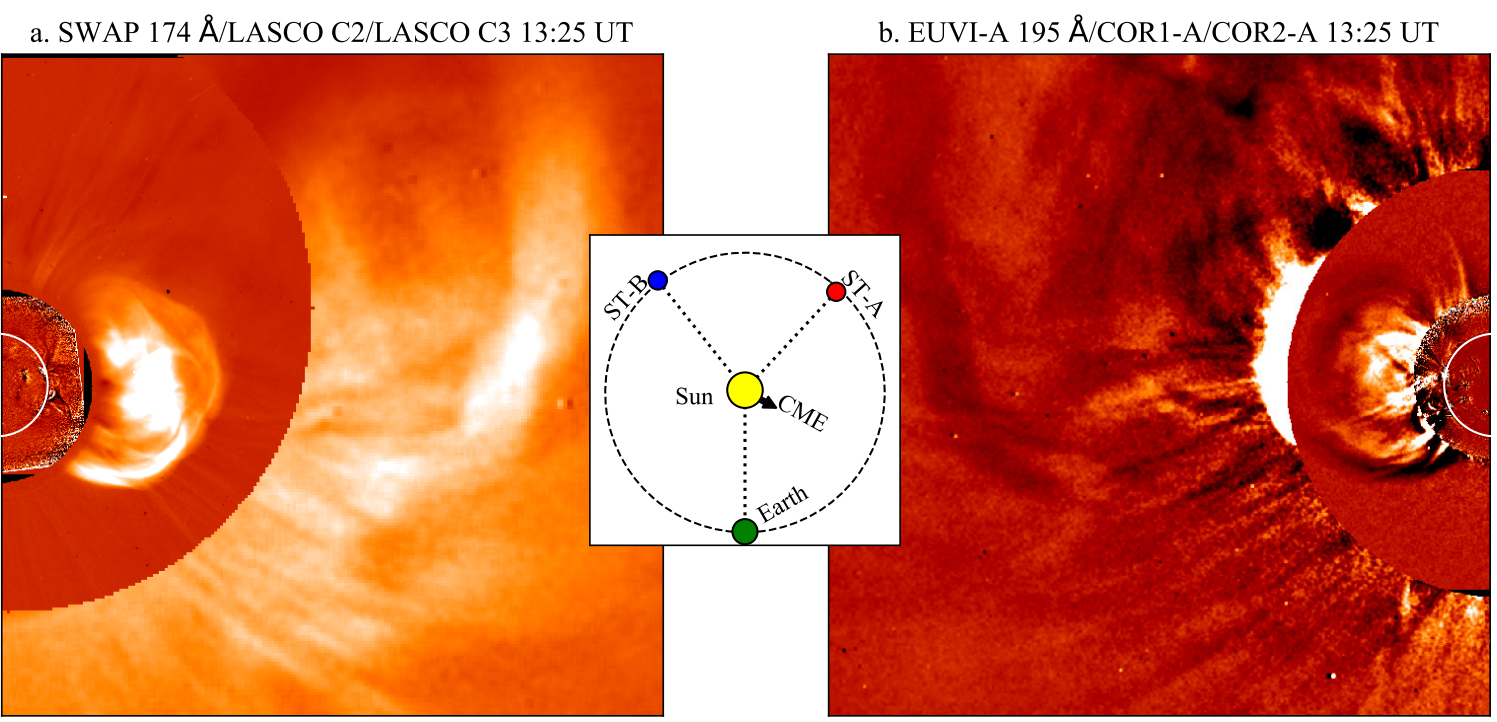

Fig. 3. Coronal mass ejections observed on 22 May 2013 from Earth and STEREO-A. (a) The CMEs observed on 22 May 2013 from Earth's perspective in a composite image from SWAP $174 \AA$ A, LASCO/C2, and LASCO/C3. The CME associated with the radio emission in Fig. 2 can be seen in the LASCO/C2 field of view (inner), while the earlier CME has expanded higher up in the corona and can be seen in the LASCO/C3 field of view (outer). (b) The CMEs observed on 22 May 2013 from STEREO-A's perspective in a composite image from EUVI-A 195 A, COR1-A, and COR2-A. The main CME is visible in the COR1-A field of view (inner), while the earlier CME is visible in the COR2-A field of view (outer). The middle inset in the figure shows the propagation direction of the CME and the location of Earth and the STEREO spacecraft with respect to the Sun.

evolution of the main CME in the low corona observed by SWAP and both CMEs in the high corona observed by COR2-A can be seen in Movies 2 and 3 online, respectively. The preceding
CME was first observed in white-light images from COR1-A at 09:24 UT, occurring $\sim 4$ hours before the main CME studied here. Therefore, the main CME, which occurred at 13:00 UT, 


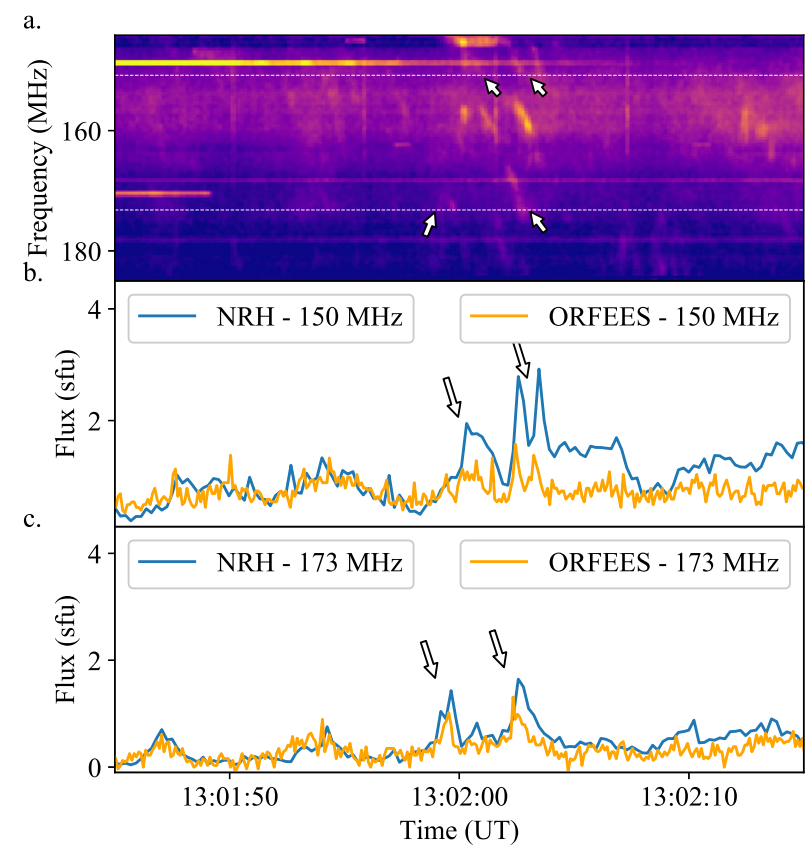

Fig. 4. Spectral features corresponding to the moving radio burst labelled as Source 2 in Fig. 1. Figures corresponding to Sources 1, 2, and 3 are included in Appendix A. (a) Zoomed-in view of the ORFEES dynamic spectrum showing a 30-s period starting at 13:01:45 UT. The first arrow points at one of the Source 2 bursts shown in the middle-left panel of Fig. 1. $(b-c)$ Time series of the NRH flux density of Source 2 in sfu and ORFEES normalised intensity in arbitrary units at $(b) 150$ and (c) $173 \mathrm{MHz}$.

propagated through a strongly disturbed coronal plasma consisting of material and magnetic field lines behind the earlier $\mathrm{CME}$. The main CME propagated through the outer corona with a de-projected speed of $\sim 1500 \mathrm{~km} \mathrm{~s}^{-1}$, as reported by Palmerio et al. (2019), and eventually caught up with the preceding CME. LASCO/C3 observations show the CMEs merging at an altitude of $\sim 20 R_{\odot}$ (reported by Palmerio et al. 2019, based on plane-of-sky images). The resulting ejecta was observed in situ at STEREO-A, while the interplanetary shock driven by the merged structure was detected at both Earth and STEREO-A (Palmerio et al. 2019).

\section{Results}

The moving radio bursts presented in Fig. 1 are observed at the two lowest $\mathrm{NRH}$ frequencies, 150 and $173 \mathrm{MHz}$, and do not show any circular polarisation. In order to identify the possible emission mechanism, origin of the energetic electrons generating this emission, and their relation to the CME we examine the spectral characteristics, kinematics, and 3D location of these moving bursts.

\subsection{Spectral characteristics of the moving radio bursts}

A detailed dynamic spectrum shows Source 2 for a period of 30 s starting from 13:01:45 UT (Fig. 4a). Similar figures, corresponding to Sources 1 and 3, together with an additional example for Source 2, are shown in Appendix A. The Type I noise storm is the dominant emission in the ORFEES dynamic spectra shown in Figs. 4a and in the figures in Appendix A. However, at times when the moving radio bursts appear in NRH images, they are sometimes brighter than the dominating noise storm. The moving sources correspond to individual bursts that are separate from the noise storm in the ORFEES dynamic spectra.

To verify the association between the features observed in ORFEES spectra and the moving radio sources, we compute the flux densities of the moving bursts and compare them to the corresponding ORFEES time series. The flux densities of the moving radio bursts observed by $\mathrm{NRH}$ are estimated at 150 and $173 \mathrm{MHz}$ for all three sources. The flux densities are estimated inside a zoomed-in box covering the full extent and movement of the radio source, and they include the pixels with levels $>20 \%$ of the maximum intensity levels in each box. We choose a threshold of $20 \%$ to include the full extent of the radio source and exclude quiet-Sun or other types of weak emission. The flux densities are estimated in solar flux units (sfu; where $1 \mathrm{sfu}=10^{-22} \mathrm{~W} \mathrm{~m}^{-2} \mathrm{~Hz}^{-1}$ ) for total intensity (Stokes I). The NRH flux densities (blue time series in Figs. 4b-c at 150 and $173 \mathrm{MHz}$ ) show peaks of bursty emission. Similar behaviour is shown in the ORFEES normalised intensities at 150 and $173 \mathrm{MHz}$ (orange time series in Figs. $4 \mathrm{~b}-\mathrm{c}$ ) that are extracted from the dynamic spectrum in Fig. $4 \mathrm{a}$.

The bursts marked by the white arrows in the top panel of Fig. 4 correspond to the moving radio burst that we labelled as Source 2. In particular, the burst in Fig. 4 marked by the leftmost white arrow corresponds to the NRH burst in the left middle panel of Fig. 1. We note that there is a slight delay of $\sim 0.5 \mathrm{~s}$ between the NRH and ORFEES time stamps. We also note that ORFEES is less sensitive than the NRH, which may be the reason why it does not observe the moving radio sources when they are fainter compared to the examples shown in Fig. 4 and Appendix A. The majority of bursts identified in the ORFEES spectrum that correspond to the moving radio bursts (including those presented in the figures in Appendix A) represent fine-structured, narrow-band bursts. These bursts have forward or reverse drifts and durations of $\sim 1 \mathrm{~s}$. Thus, these drifting bursts resemble herringbones. However, they occur on their own instead of occurring in large clusters and they also do not show the presence of a 'backbone' with forward and reverse drift bursts on either side (Morosan et al. 2019a). Similarly to what was recently reported by Magdalenić et al. (2020), other bursts somewhat resemble the fine structures composing Type II bursts (see Fig. A.2). The morphological resemblance of drifting bursts and herringbones indicates that also the emission mechanism of both types of bursts could be the same, that is, plasma emission. In such a scenario, the accelerated electron beams, associated with drifting bursts, would travel in various directions, similar to the way herringbones are generated (Holman \& Pesses 1983; Cairns \& Robinson 1987). However, we note that there are also other potential sources to these electron beams than acceleration at the CME shock (see the Introduction). The knowledge on the spatial location of these fine-structured bursts will provide some additional insight into the origin of these bursty electron beams, which is further investigated in the following sections.

\subsection{Moving radio sources and CME kinematics}

The moving radio sources are observed at unusually high altitudes (up to $2.2 R_{\odot}$ in Fig. 1) for emission at 150 and $173 \mathrm{MHz}$. In the background solar corona, a plasma frequency level of $150.9 \mathrm{MHz}$ corresponds to a height range of $1.05-1.35 R_{\odot}$ for fundamental emission and $1.20-1.65 R_{\odot}$ for harmonic emission, based on electron density models of the corona (Newkirk 1961; Saito et al. 1977). The centroids of the radio emission at $150.9 \mathrm{MHz}$ show outward movement in the direction of the CME expansion (Fig. 5). Source 1 and Source 3 move in a 

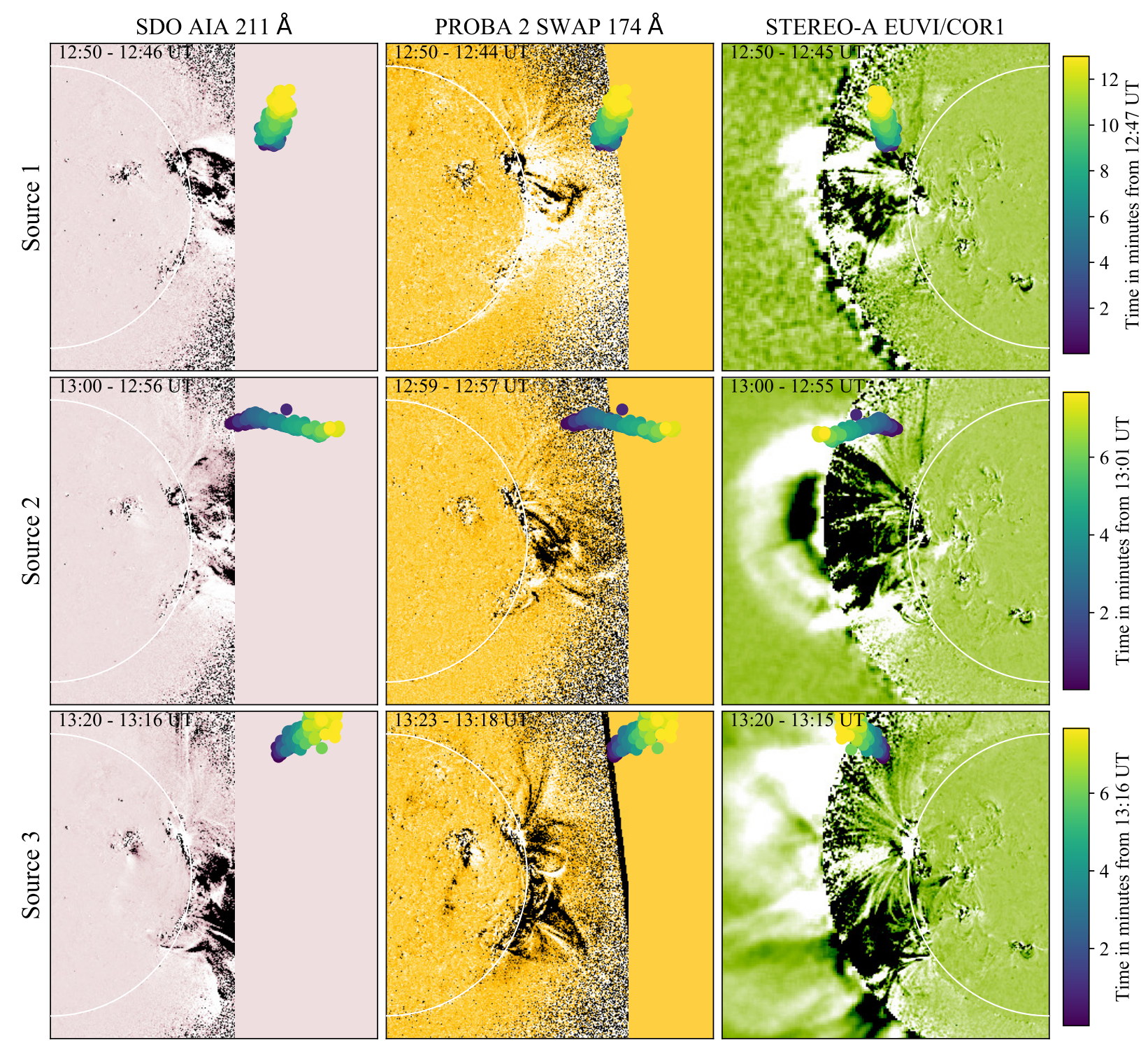

Fig. 5. Evolution of the centroids of the moving radio sources through time. The centroids of the three moving sources are shown from top to bottom overlaid on SDO/AIA (left), PROBA2/SWAP (middle), and STEREO/EUVI-A and COR1-A (right) running-difference images of the Sun. The colour scale for the centroids represents minutes from the start time of each moving radio burst. The bursts are located at the northern CME flank in both the Earth (SDO and PROBA2) and STEREO-A perspectives.

north-westerly direction, along with the CME flank, while Source 2 moves in a westerly direction with the CME apex. However, from Earth's viewpoint, this plane-of-sky movement can be affected by line-of-sight projection effects. While a significant portion of this movement occurs close to the plane of sky, the sources could also be directed at an angle towards or away from the observer. We thus aim to reconstruct a 3D perspective using CME observations from STEREO-A that was at an ideal location to provide a view of the eruption from the other side (see the inset in Fig. 4a). STEREO-A provides observations in both extreme ultra-violet (EUV) and white light, extending to larger heights than SDO or PROBA2 (the STEREO/COR1 field of view extends between 1.5 and $4 R_{\odot}$ ).

To determine the position of the radio sources in relation to the two CMEs in the STEREO-A perspective, we de-project the radio source centroids from their plane-of-sky view. Assuming that the radio bursts are emitted close to the plane of sky (i.e. the $z$-coordinate of this emission is $\sim 0$ ), it is possible to estimate their approximate location in the STEREO-A perspective.
The $z$-coordinates, combined with the plane-of-sky coordinates of the NRH centroids, are then used to project the coordinates of the radio sources onto the STEREO-A plane and to also represent them in $3 \mathrm{D}$. The assumption that the $z$-coordinate of this emission is $\sim 0$ can be used since the centroids show significant movement in the plane of sky. However, we note that the radio sources could also move towards or away from the observer, from Earth's perspective, and only cross the plane of sky as they propagate. Moreover, the centroids could also be located away from the plane of sky. However, if we consider a large and negative $z$-coordinate, then the centroids would be located too far away from the CME flank in the STEREO-A perspective. If we consider a large and positive $z$-coordinate, then the centroids would be located farther out, close to the CME apex and at even higher altitudes, which is unrealistic for plasma emission. The $z$-coordinate of the radio centroids most likely has values in the range $\pm 300^{\prime \prime}$, which we assign as the uncertainty range for our de-projection method. This uncertainty range is also consistent with the 3D location of the main CME. Therefore, the 


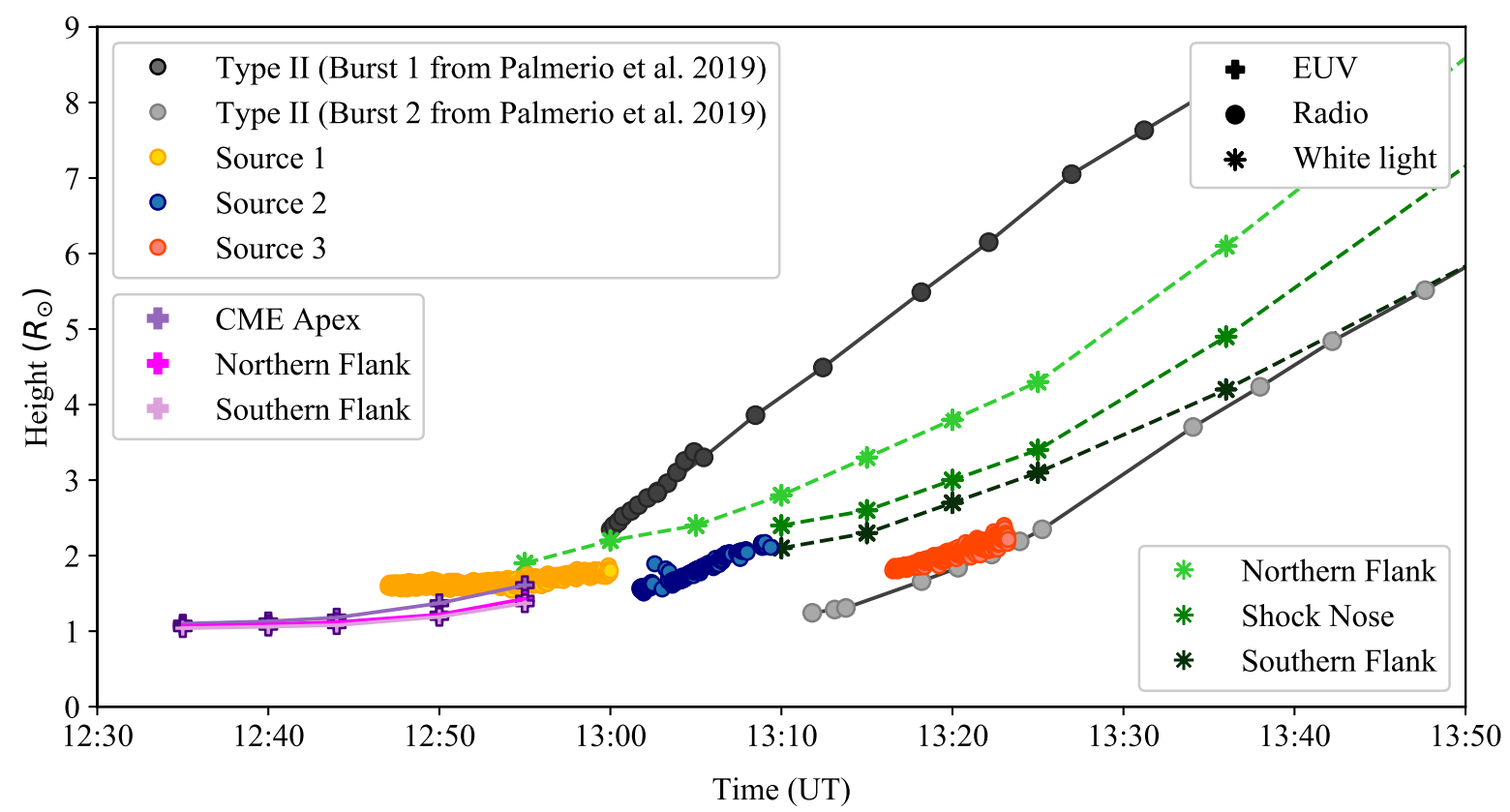

Fig. 6. Kinematics of the moving radio bursts and Type II lanes in relation to the CME expansion. The Type II bursts and CME kinematics were previously studied by Palmerio et al. (2019). The figure is adapted from Palmerio et al. (2019), to which we included the three moving radio sources studied here. The EUV measurements are taken with PROBA2/SWAP and STEREO/EUVI-A, the white-light ones with LASCO/C2-C3 and STEREO/COR1-COR2-A, and the radio ones with NDA and the WAVES instruments onboard Wind (Ogilvie \& Desch 1997) and both STEREO spacecraft.

assumption that the radio sources propagate close to the plane of sky is a good approximation to determine their location in other perspectives, and relative to the two associated CMEs.

The radio centroids of all three sources (coloured with progressing time in Fig. 5), show the propagation of the radio bursts. The radio sources are superposed on SDO/AIA $211 \AA$ images (left), PROBA2/SWAP $174 \AA$ images (middle), and STEREOA combined EUVI (at $195 \AA$ ) and COR1 images (right). Both Earth (AIA and SWAP) and STEREO perspectives show that the radio sources are located close to the northern CME flank. At the northern flank, the bent field lines due to the passage of the previous CME can be observed in EUV and white-light images (see Figs. 3 and 5). Despite the uncertainty associated with our de-projection technique, the used method would not affect the kinematics of the moving radio bursts in the STEREO perspectives, and these radio bursts would still be associated with the northern CME flank. The unusually high altitudes of these radio bursts may be related to the fact that the CME expands through a strongly disturbed corona.

The plane-of-sky centroid locations can be compared to the CME kinematics. The CME kinematics have already been studied in relation to the Type II lanes observed at lower frequencies by Palmerio et al. (2019). Unfortunately, imaging at lower frequencies is unavailable for this event and an electron densixty model was used to extract the heights of the Type II lanes observed by NDA and STEREO S-WAVES shown in Fig. 2. The kinematics of the CME in relation to the Type II lanes is shown in Fig. 6, adapted from Palmerio et al. (2019), including the additional three moving radio bursts analysed in this study. The early Type II (labelled as Burst 1 in Palmerio et al. 2019) is faster than the associated CME, while the later Type II (labelled as Burst 2 in Palmerio et al. 2019) appears to match the southern shock flank kinematics deduced from white light. The plane-ofsky centroids of the moving radio bursts are shown in yellow (Source 1), blue (Source 2), and red (Source 3). Source 2 appears to match the white-light shock nose propagation despite being located at the northern CME flank. This, however, agrees with the propagation direction of the Source 2 centroids in Fig. 5, that appear to travel radially outwards in the direction of the CME apex, and not laterally outwards with the CME flank. On the other hand, Source 3 has a similar slope to the northern flank shock expansion, which again agrees with the propagation direction of the centroids in Fig. 5. Source 1 cannot be as clearly related either to the CME flanks or CME apex, since both the apex and CME flanks have similar propagation speeds in the early phase of the eruption, however, as shown in Fig. 5, it has a similar propagation direction to Source 3. The planeof-sky speeds of these sources are $\sim 400 \mathrm{~km} \mathrm{~s}^{-1}, \sim 1500 \mathrm{~km} \mathrm{~s}^{-1}$, and $\sim 950 \mathrm{~km} \mathrm{~s}^{-1}$ for Sources 1, 2, and 3, respectively. We note that Sources 2 and 3 are particularly fast, and have speeds similar to those of Type II bursts (Mancuso \& Raymond 2004; Palmerio et al. 2019). Source 1 appears to propagate much slower in the plane of sky, however, we note that the CME speed obtained from plane-of-sky EUV observations was also slow during its early stages (see the EUV measurements in Fig. 6). It should be also noted that estimated plane-of-sky speeds represent a lower limit since it is possible that these sources also propagate towards or away from the observer, therefore all radio sources could be propagating faster and have steeper slopes than those shown in Fig. 6.

\section{3. $3 D$ reconstruction of the locations of electron acceleration}

The three perspectives provided by SOHO and the two STEREO spacecraft allow us to reconstruct the 3D structure of the CME evolving through the solar corona. We use the Graduated Cylindrical Shell (GCS; Thernisien et al. 2006, 2009) model, which consists of a parameterised croissant-shaped shell, to manually trace the CME through time in nearly-simultaneous coronagraph 

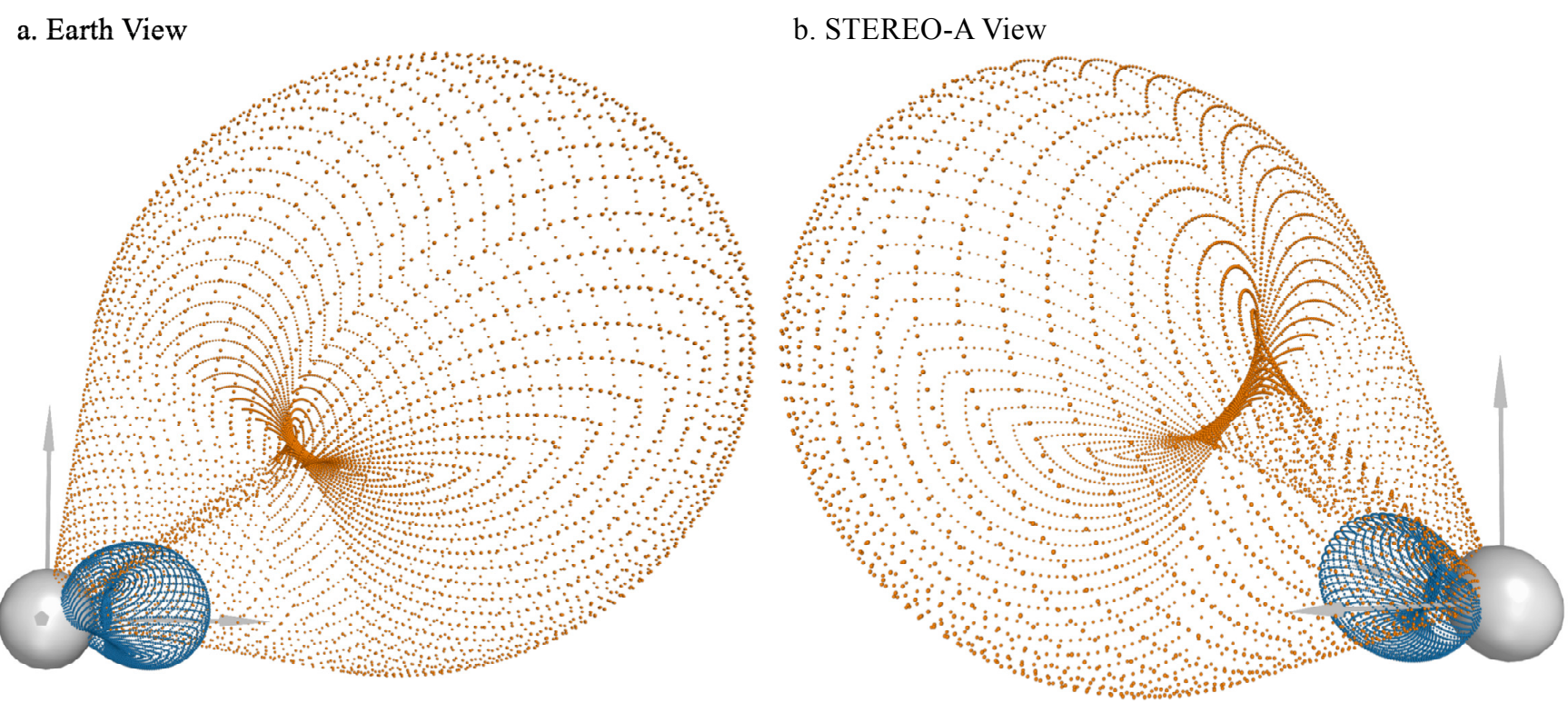

Fig. 7. CME expansion in 3D relative to the earlier ongoing CME. The main CME is shown as a wireframe consisting of blue dots, while the ealier CME is shown as an orange wireframe. Two viewpoints are shown from $(a)$ Earth's perspective and $(b)$ STEREO-A's perspective.

Table 1. CME parameters derived from the GCS reconstructions.

\begin{tabular}{lcccc}
\hline & Long & Lat & Tilt & Speed \\
\hline Earlier CME & $73^{\circ}$ & $32^{\circ}$ & $-48^{\circ}$ & $663 \mathrm{~km} \mathrm{~s}^{-1}$ \\
Main CME & $67^{\circ}$ & $7^{\circ}$ & $-28^{\circ}$ & $1160 \mathrm{~km} \mathrm{~s}^{-1}$ \\
\hline
\end{tabular}

Notes. Longitudes and latitudes are provided in Stonyhurst coordinates, and the tilt is defined positive for counterclockwise rotations.

images. We can then model the observed $\mathrm{CME}$ in $3 \mathrm{D}$ by overlaying the parametrised shell onto the three planes of sky (SOHO, STEREO-A, and STEREO-B). We perform reconstructions from 13:05 to 13:30 UT (at the time of STEREO images) at five-minute intervals during the early propagation of the CME. Since the CME appeared in the LASCO/C2 field of view at 13:25 UT, we perform reconstructions using only the COR1-A and $-\mathrm{B}$ viewpoints from 13:05 to 13:20 UT that are guided by the CME parameters obtained from a later time when all three viewpoints are available. Furthermore, we also perform reconstructions of the earlier CME around the same times. The CME reconstruction includes only the CME cavity, excluding the surrounding white light shock.

The reconstructed CMEs are shown in Fig. 7 from the Earth (left) and STEREO-A (right) perspectives, as wireframes consisting of blue dots for the main CME and orange dots for the earlier CME. The reconstructed parameters of the two CMEs, such as propagation longitude, latitude, tilt (i.e. axis orientation), and speed are given in Table 1. Although the two CMEs originate from the same source region (AR 11745), they have different propagation directions. The main CME expands into the earlier CME mainly with its northern portion, while the southern flank expands through the less disturbed background corona. The 3D reconstruction and coronagraph images show that the main part of the earlier CME (i.e. the classic 'three-part structure' comprising of a bright outer rim, a dark cavity corresponding to the flux rope, and a bright core; Illing \& Hundhausen 1985) is already at a significant altitude when the main CME is still close to the Sun (blue wireframe). Therefore, the main
CME most likely propagates partially into the legs of the previous $\mathrm{CME}$. The magnetic field configuration of $\mathrm{CME}$ legs are usually approximately radial, however they have an additional twist component throughout their volume. While such twist is a common feature of CME models and simulations (e.g. Linker et al. 2003; Isenberg \& Forbes 2007; Aulanier et al. 2012; Lynch et al. 2016; Török et al. 2018) there is only indirect evidence of it in solar observations (e.g. Wang \& Hess 2018) and insitu observations may be difficult to interpret (e.g. Owens 2016; Al-Haddad et al. 2019). The legs can therefore be considered as 'open field lines' from the perspective of the main CME. The medium through which the CME propagates also appears significantly more disturbed at its northern flank than at its southern flank (see Figs. 3 and 5), which agrees with our 3D reconstruction of the geometry of the two CMEs. Bent coronal structures that may correspond to the legs of the earlier CME are indeed visible at a slightly later time in coronagraph images (see e.g. the features at the northern flank in Fig. 3a).

The CME reconstructions can be used to visualise the location of the associated accelerated electrons in 3D, using the $x$, $y$, and $z$-coordinates of the radio emission centroids obtained in the previous section. The moving radio sources are shown relative to the two CMEs in 3D in Fig. 8. This figure represents a zoomed-in version of the perspectives in Fig. 7. The three moving radio sources are shown from top to bottom, and from three viewing angles: Earth (left panels), STEREO-A (middle panels), and a view from the solar North Pole (right panels). The moving radio source centroids are assumed to occur in or close to the plane of sky as outlined in the previous section. Nevertheless, we note that these radio bursts could also move away or towards the observer from Earth's viewpoint, which would translate to a movement towards Earth or a movement towards STEREO-A, respectively, in the North Pole viewpoint shown in Fig. 8. Studies of the Type II bursts associated with the main CME have found that the Type II emission is directed towards STEREOA (see Fig. 2 from Mäkelä et al. 2016). It is possible that the moving bursts studied here are also likely to be directed towards STEREO-A, away from the plane of sky, in the direction of the CME shock front expansion. The moving radio bursts positions, 

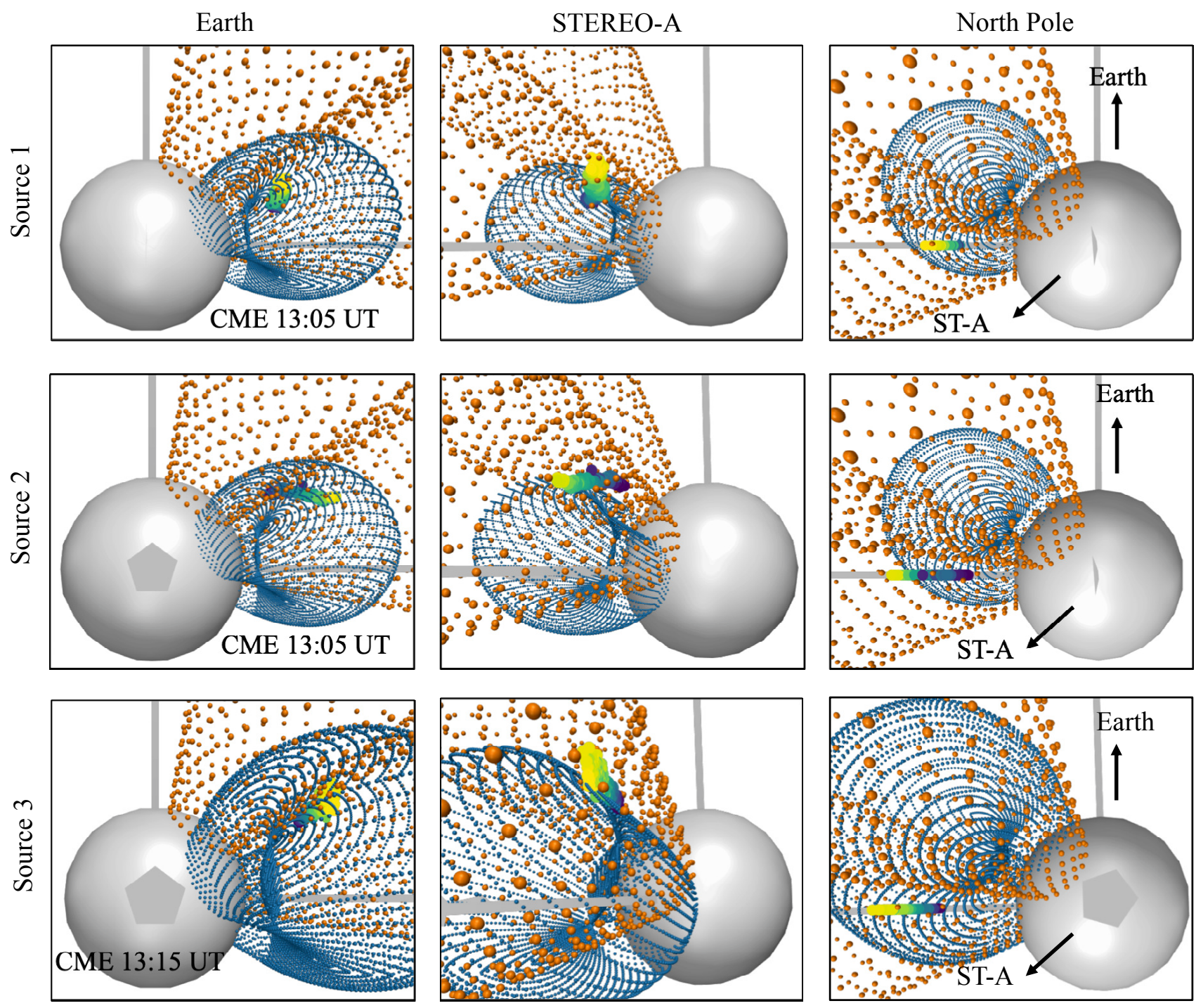

Fig. 8. CME expansion in 3D together with the associated radio emission locations. The main CME is shown as a wireframe consisting of blue dots, while the ealier CME is shown as an orange wireframe. Three viewpoints are shown, i.e. (left) Earth's perspective, (middle) STEREO-A's perspective, and (right) the solar North Pole.

however, are a good representative as to where the radio bursts are located with respect to the two CMEs.

All three moving radio sources appear to be located outside the reconstructed main $\mathrm{CME}$ bubble (blue wireframe) and at the same time inside the reconstructed CME bubble of the earlier CME (orange wireframe). Although the three moving sources are located outside the CME structure, they could still be associated to a shock or waves propagating ahead of the CME, which were not included in the wireframe reconstruction. Since the $z$-coordinate of the centroids is most likely located within a fixed range close to the plane of sky, the centroid locations at the CME flank and inside the earlier CME remain unaffected by the uncertainty in the $z$-coordinate. The times of the CME reconstructions labelled in Fig. 8 are approximately during the mid-time of the propagation of the radio source centroids (which have the same colouring through time as the colour bar in Fig. 5) for Sources 2 and 3. In the case of Source 1, the CME reconstruction time is $\sim 18 \mathrm{~min}$ after the onset of the radio emission, since we were unable to perform a reconstruction before 13:05 UT due to the CME not fully appearing in STEREO/COR1-A (the first appearance at 12:55 UT reported in Sect. 2 indicates the very leading edge of the CME, which is not enough to perform a full 3D reconstruction). Nevertheless, the $3 \mathrm{D}$ reconstructions show that all moving bursts are associated with the northern CME flank and they are located outside the main CME flank and inside the earlier CME. The 3D reconstruction also agrees with the kinematics in Fig. 6, where Source 1 and 3 propagate laterally outwards with the northern CME flank, while Source 2 does not show a northern propagation direction but only a radially outwards one, close to the propagation direction of the CME apex.

\section{Discussion}

We have so far shown that bursty signatures of electron beams originate at the northern CME flank, which expands in the wake of an earlier CME. This radio emission closely follows the CME propagation. We believe that two successive CMEs created favourable conditions for the acceleration of electron beams that generated the radio emission observed at such large heights.

To better understand the origin of energetic electrons producing this high-altitude bursty emission, we explore in more detail the coronal densities and magnetic field environment 

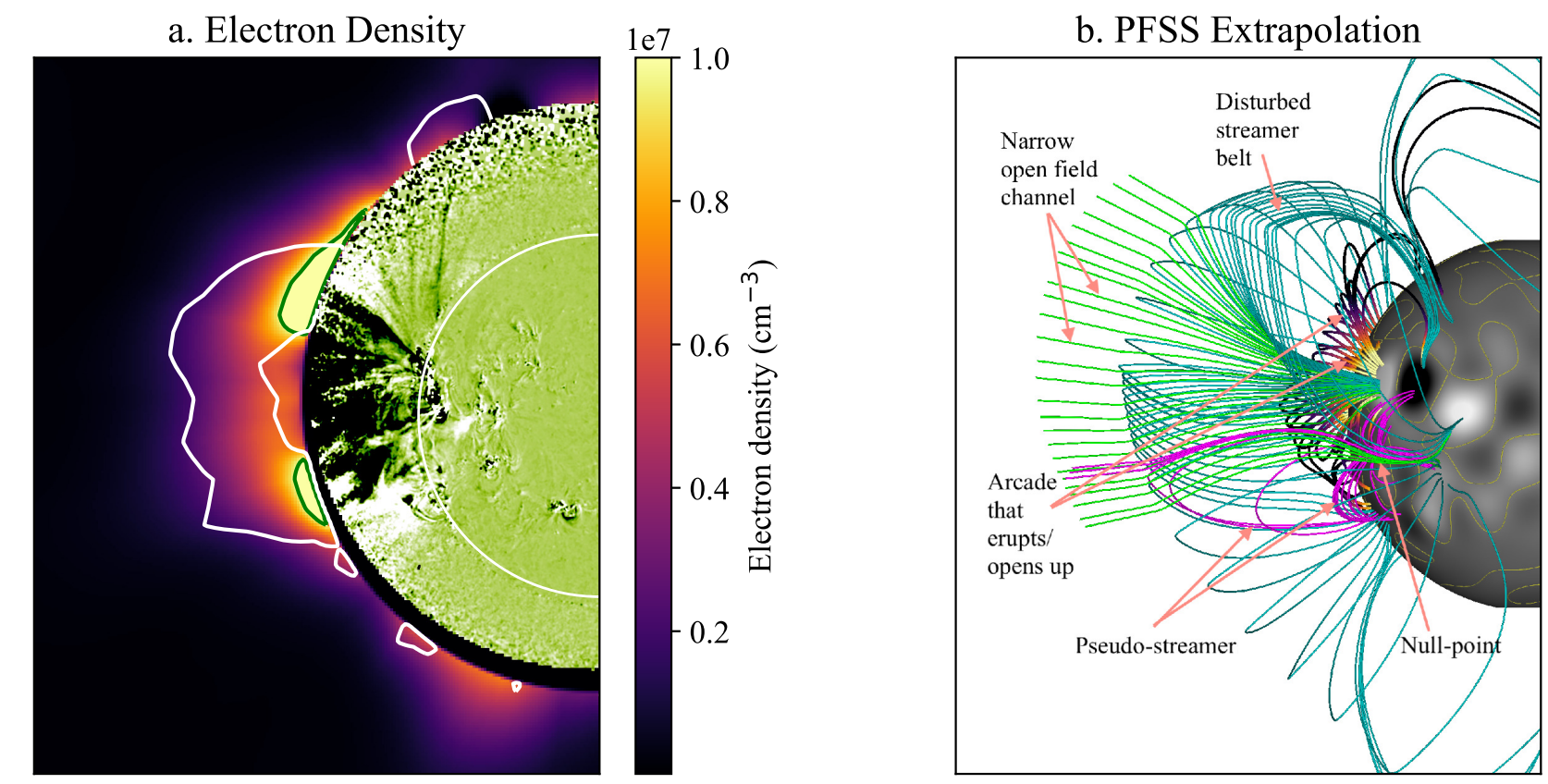

Fig. 9. Electron density and magnetic field environment through which the CME propagates. (a) Electron density map of the solar corona from the STEREO-A perspective. The green contours represent the $10^{7} \mathrm{~cm}^{-3}$ density level and the white contours outline the CME density structure after a background map has been subtracted. (b) PFSS extrapolation from the photospheric magnetic field showing the coronal flux systems in the vicinity of the CME source region.

through which the CME propagates with its northern flank. Plane-of-sky electron densities in the corona can be obtained from measurements of polarised brightness recorded by coronagraphs (van de Hulst 1950). The CME was first observed by STEREO/COR1-A, which can be used to construct electron density maps during its eruption, using the spherically symmetric polynomial inversion technique of Wang \& Davila (2014), which is based on the assumption that the radial electron density distribution has a polynomial form. An example of such a map is shown in Fig. 9a, where the electron densities in $\mathrm{cm}^{-3}$ obtained from COR1-A are shown in the outer field of view together with an EUVI-A image of the CME at 13:00 UT in the inner field of view for comparison. The white contours denote the outline of the CME in the density map after a background map is subtracted. The green contours denote the $10^{7} \mathrm{~cm}^{-3}$ density level. The moving radio bursts start propagating before the interface between the EUVI and COR1 fields of view and, in the case of Source 2 and 3, the sources propagate into the COR 1 field of view before they disappear, assuming this emission occurs close to the plane of sky. A small region of enhanced densities $\left(>10^{7} \mathrm{~cm}^{-3}\right)$ at the northern CME flank in Fig. 9a coincides with the location of the high-altitude radio emission which also maps a high density structure. Densities $>10^{7} \mathrm{~cm}^{-3}$ are significantly larger than those predicted by density models of the background corona such as the Saito et al. (1977) model or the Newkirk (1961) model, which would place these densities at a height of up to $1.35 R_{\odot}$. The electron density map places these densities as high as $1.7 R_{\odot}$. Such a big active region is also likely to be accompanied by enhanced densities radially outwards. We note that such density maps, as presented in Fig. 9a, are useful in providing a global plane-of-sky picture of the coronal electron densities. Densities of the order of $10^{7} \mathrm{~cm}^{-3}$ and the large heights of the radio emission observed correspond to harmonic plasma emission, since harmonic emission at a frequency of $150 \mathrm{MHz}$ would originate at a plasma electron number density equal to $7 \times 10^{7} \mathrm{~cm}^{-3}$.
Models of the coronal magnetic field can be used to better understand the CME eruption and propagation through the ambient corona and its magnetic field. A selection of representative magnetic field lines associated with the flux systems in the vicinity of the CME eruption is shown in Fig. 9b, from the STEREO-A perspective. The Fig. 9b field lines are derived from the Potential Field Source Surface (PFSS; e.g. Wang \& Sheeley 1992; Schrijver \& De Rosa 2003) extrapolation based on photospheric magnetograms observed by the National Solar Observatory (NSO) Global Oscillation Network Group (GONG; Harvey et al. 1996). These field lines are a good representation of the ambient background magnetic field prior to the main CME. Close to the northern flank region, in white-light observations, we also observed the legs of the preceding CME, which can be categorised as open field with an additional twist component that would be present in addition to the PFSS field lines.

The field lines coloured from purple to yellow in Fig. 9b show the arcade above the polarity inversion line of the active region that either erupts to form the CME or opens up following the CME eruption. After eruption, the main CME propagates through a disturbed helmet streamer belt (blue field lines) at its northern flank, which changes from a north-south direction above the active region to an east-west direction north of the active region. A narrow channel of open flux is denoted by the green lines. At the southern flank, a coronal null point and pseudostreamer are present, denoted by the magenta field lines. Coronal loops that are arranged consistently with the nullpoint and pseudostreamer geometry can also be observed in SWAP $174 \AA$ images of the Sun before the CME eruption (see Movie 2 online). The radio emission originates at the northern flank, where the main CME appears to laterally expand into the closed flux system of the disturbed helmet streamer belt and the 'open' field lines of the legs of the preceding CME. The pseudostreamer null point, and thus the region where magnetic reconnection would occur following the eruption, is located at the southern flank instead. Well after our three northern flank 
sources occur, other stationary bursts seem to indeed originate from the southern flank pseudostreamer null point in the wake of the main CME eruption, as seen in plane-of-sky images (see Movie 1 online).

The moving radio bursts propagate outwards with the northern flank of the main CME through the magnetic field lines, strongly disturbed plasma, and possible enhanced density structures left behind by the earlier CME. An early interaction between these two CMEs is most likely the cause of this emission that shows fine structures with both forward and reverse drifts indicating individual electron beams propagating in various directions. Similar signatures of electron beams have previously been observed as herringbones, when the CME drives a shock predominantly at the flank (Cairns \& Robinson 1987; Morosan et al. 2019a). However, in this case Type II emission occurs at lower frequencies during the onset of Sources 2 and 3. Thus, a shock is present in the corona at least during the occurrence of Sources 2 and 3 but it may not be the only element responsible for this emission.

Collapsing magnetic traps have been proposed by Magdalenić et al. (2002) as a mechanism to accelerate electrons to explain the occurrence of Type II bursts and herringbones in the low corona, where shocks originate during the early stages of an eruption with low Mach numbers (Kumari et al. 2019), making them inefficient particle accelerators. Figure 10a is a schematic for possible interactions of the main CME with the magnetic field and plasma of the disturbed corona left behind by the previous CME. These interactions, illustrated in Figs. 10b and 10c, are favourable conditions for the creation of collapsing magnetic traps. In this scenario, electrons can reflect at mirror points created by a shock wave intersecting magnetic field lines twice. The electrons can thus reflect several times before they become energised and escape in directions towards or away from the Sun, to generate the radio emission observed. One way for the CME-driven shock wave to intersect the open field lines twice is to have a wavy or turbulent shock front, as shown in Fig. 10b. An alternative, shown in Fig.10c, has curved or helical open magnetic field lines upstream of a planar shock (e.g. Sandroos \& Vainio 2006). The twist in the magnetic field in the legs of the earlier CME could create such conditions.

Theoretical studies predict that particles accelerated in magnetic traps will gain more parallel momentum, eventually being able to escape the trap, however with a small energy gain (Sandroos \& Vainio 2006). The small energy gain suits our observations since the moving radio bursts observed consist of weak and short-lived radio emission compared to the other main radio bursts present (Type IIs and Type IVs). These temporary collapsing traps are also expected to move outwards with the CME expansion, therefore the radio bursts would show an outwards movement through time. The presence of a shock wave in the low corona at the time of the moving radio bursts is already indicated by the ongoing Type II emission lanes observed at lower frequencies. We also note that due to the presence of a disturbed streamer that has an east-west orientation at the northern flank (Fig. 9b), the CME propagates quasi-perpendicularly to the overlying magnetic field, making it more likely for particles to be accelerated at a $\mathrm{CME}$ shock front at this location. However, the presence of a CME-driven shock is not a necessary condition, since any CME-driven wave can intersect the ambient field lines in a similar manner. Such a mechanism can explain the presence of these unusual high-altitude radio bursts predominantly at the northern flank, their frequency drift, and their limited duration, since these collapsing traps disappear as the CME evolves and propagates away from the Sun.
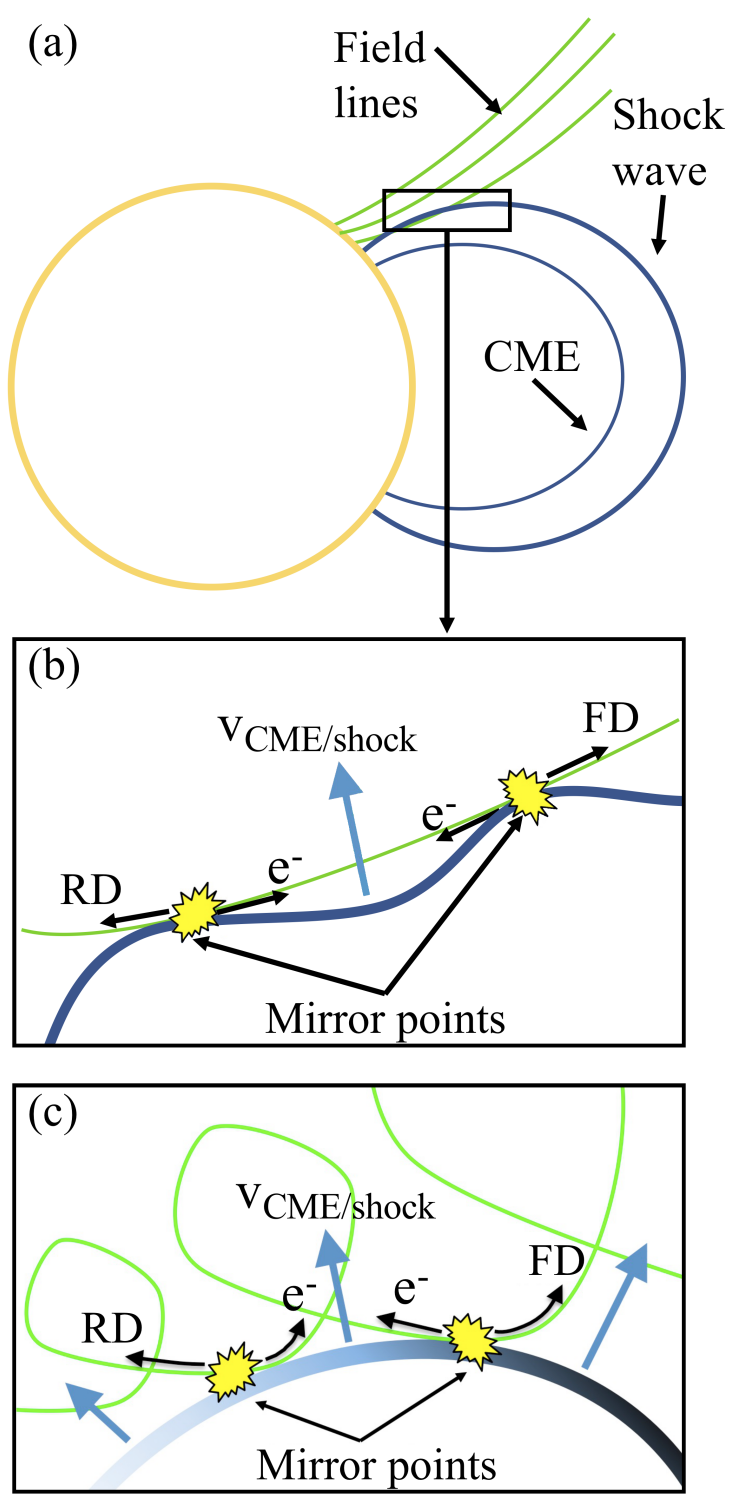

Fig. 10. Schematic demonstrating the generation of the magnetic trap geometry. This schematic shows how the CME-CME or CMEbackground field interaction can generate collapsing magnetic trap geometries likely to generate the moving bursts studied here (after Magdalenić et al. 2002). When the CME-driven shock wave intersects an open field line twice, these intersections form two mirror points as the CME front advances in the direction indicated by $v_{\mathrm{CME} / \text { shock }}$. At the mirror points, electrons $\left(\mathrm{e}^{-}\right)$can bounce back and forth until they escape. Once the electrons escape they can produce forward drift (FD) and reverse drift (RD) radio bursts. This can arise via a wavy shock front intersecting radial open fields (panel $b$ ) or a planar shock front intersecting helical open field lines (panel $c$ ).

\section{Conclusion}

We observed for the first time bursty emission originating close to the CME flank region, which is not included in the standard classification of solar radio bursts. A likely mechanism that we propose as responsible for generating the bursty electron beams are collapsing magnetic traps. These traps are favoured by the complex ambient coronal conditions created by two interacting CMEs. Our observations show that the conditions and mechanism of particle acceleration during the CME eruption are highly dependent on the ambient plasma characteristics and complexity of the magnetic field through which the CME propagates. The 
collapsing magnetic trap mechanism may also be important for space weather, since it allows the acceleration of fast electrons even in weak CME-driven shocks. Collapsing magnetic traps are also a universal acceleration mechanism, since they can be found in other plasmas such as planetary magnetospheres and bow shocks (e.g. Gisler \& Lemons 1990).

Our study demonstrates that the additional perspectives provided by the STEREO mission were necessary to determine the nature of the moving radio bursts. $3 \mathrm{D}$ reconstructions of the eruptions were essential to show how the two CMEs propagate with respect to each other. Future observations of moving radio bursts associated with CMEs, combined with possibly additional vantage points from the STEREO-A spacecraft and future L5 missions, should be used to better understand the numerous possibilities for particle acceleration during CME eruptions. Such studies also have the potential to update the current classification of solar radio emission.

Acknowledgements. The results presented here have been achieved under the framework of the Finnish Centre of Excellence in Research of Sustainable Space (Academy of Finland grant number 312390), which we gratefully acknowledge. E. P. acknowledges the NASA Living With a Star Jack Eddy Postdoctoral Fellowship Program, administered by UCAR's Cooperative Programs for the Advancement of Earth System Science (CPAESS) under award no. NNX16AK22G. E. K. J. K. acknowledges the ERC under the European Union's Horizon 2020 Research and Innovation Programme Project SolMAG 724391, and Academy of Finland Project 310445. J. M. acknowledges funding by the BRAIN-be (Belgian Research Action through Interdisciplinary Networks) project CCSOM (Constraining CMEs and Shocks by Observations and Modelling throughout the inner heliosphere). B. J. L. acknowledges support from NASA HGI 80NSSC18K0645, LWS 80NSSC19K0088, and NSF AGS 1851945. M. P. acknowledges the European Research Council (ERC) Consolidator grant 682068-PRESTISSIMO, and Academy of Finland grants 312351 and 309937. We thank the Radio Solar Database service at LESIA \& USN (Observatoire de Paris) for making the NRH/NDA/ORFEES data available.

\section{References}

Al-Haddad, N., Poedts, S., Roussev, I., et al. 2019, ApJ, 870, 100 Aulanier, G., Janvier, M., \& Schmieder, B. 2012, A\&A, 543, A110

Bastian, T. S., Pick, M., Kerdraon, A., Maia, D., \& Vourlidas, A. 2001, ApJ, 558, L65

Boischot, A. 1957, Acad. Sci. Paris C. R., 244, 1326

Boischot, A., Rosolen, C., Aubier, M. G., et al. 1980, Icarus, 43, 399

Bougeret, J. L., Goetz, K., Kaiser, M. L., et al. 2008, Space Sci. Rev., 136, 487

Bouratzis, C., Hillaris, A., Alissandrakis, C. E., et al. 2016, A\&A, 586, A29

Brueckner, G. E., Howard, R. A., Koomen, M. J., et al. 1995, Sol. Phys., 162 357

Cairns, I. H., \& Robinson, R. D. 1987, Sol. Phys., 111, 365

Cane, H. V., \& White, S. M. 1989, Sol. Phys., 120, 137

Carley, E. P., Long, D. M., Byrne, J. P., et al. 2013, Nat. Phys., 9, 811

Chrysaphi, N., Kontar, E. P., Holman, G. D., \& Temmer, M. 2018, ApJ, 868, 79

Chrysaphi, N., Reid, H. A. S., \& Kontar, E. P. 2020, ApJ, 893, 115

Ding, L., Jiang, Y., Zhao, L., \& Li, G. 2013, ApJ, 763, 30

Domingo, V., Fleck, B., \& Poland, A. I. 1995, Sol. Phys., 162, 1

Dulk, G. A. 1973, Sol. Phys., 32, 491

Gisler, G., \& Lemons, D. 1990, J. Geophys. Res., 95, 14925

Gopalswamy, N., Yashiro, S., Thakur, N., et al. 2016, ApJ, 833, 216

Harvey, J. W., Hill, F., Hubbard, R. P., et al. 1996, Science, 272, 1284

Holman, G. D., \& Pesses, M. E. 1983, ApJ, 267, 837

Howard, R. A., Moses, J. D., Vourlidas, A., et al. 2008, Space Sci. Rev., 136, 67

Illing, R. M. E., \& Hundhausen, A. J. 1985, J. Geophys. Res. Space Phys., 90, 275

Isenberg, P. A., \& Forbes, T. G. 2007, ApJ, 670, 1453

Kahler, S. W., \& Hundhausen, A. J. 1992, J. Geophys. Res., 97, 1619

Kaiser, M. L., Kucera, T. A., Davila, J. M., et al. 2008, Space Sci. Rev., 136, 5

Kerdraon, A., \& Delouis, J. M. 1997, in Coronal Physics from Radio and Space Observations, ed. G. Trottet (Berlin Springer Verlag), Lect. Notes Phys., 483 192
Klassen, A., Bothmer, V., Mann, G., et al. 2002, A\&A, 385, 1078 Kong, X. L., Chen, Y., Li, G., et al. 2012, ApJ, 750, 158

Kumari, A., Ramesh, R., Kathiravan, C., Wang, T. J., \& Gopalswamy, N. 2019, ApJ, 881, 24

Kwon, R.-Y., Zhang, J., \& Olmedo, O. 2014, ApJ, 794, 148

Lemen, J. R., Title, A. M., Akin, D. J., et al. 2012, Sol. Phys., 275, 17

Linker, J. A., Mikić, Z., Lionello, R., et al. 2003, Phys. Plasmas, 10, 1971

Liu, Y., Luhmann, J. G., Bale, S. D., \& Lin, R. P. 2009, ApJ, 691, L151

Liu, Y. D., Luhmann, J. G., Lugaz, N., et al. 2013, ApJ, 769, 45

Liu, Y. D., Hu, H., Zhu, B., Luhmann, J. G., \& Vourlidas, A. 2017, ApJ, 834, 158

Liu, Y. D., Zhu, B., \& Zhao, X. 2019, ApJ, 871, 8

Lynch, B. J., Masson, S., Li, Y., et al. 2016, J. Geophys. Res., 121(10), 677

Magdalenić, J., Vršnak, B., \& Aurass, H. 2002, in Solar Variability: From Core to Outer Frontiers, ed. A. Wilson, ESA Spec. Pub., 1, 335

Magdalenić, J., Vršnak, B., Zlobec, P., Hillaris, A., \& Messerotti, M. 2006, ApJ, 642, L77

Magdalenić, J., Marqué, C., Krupar, V., et al. 2014, ApJ, 791, 115

Magdalenić, J., Marqué, C., Fallows, R. A., et al. 2020, ApJ, 897, L15

Maguire, C. A., Carley, E. P., McCauley, J., \& Gallagher, P. T. 2020, A\&A, 633, A56

Mäkelä, P., Gopalswamy, N., Reiner, M. J., Akiyama, S., \& Krupar, V. 2016, ApJ, 827, 141

Mancuso, S., \& Raymond, J. C. 2004, A\&A, 413, 363

Mancuso, S., Frassati, F., Bemporad, A., \& Barghini, D. 2019, A\&A, 624, L2

Mann, G., Klassen, A., Classen, H. T., et al. 1996, A\&AS, 119, 489

Martínez Oliveros, J. C., Raftery, C. L., Bain, H. M., et al. 2012, ApJ, 748, 66

Morosan, D. E., Carley, E. P., Hayes, L. A., et al. 2019a, Nat. Astron., 3, 452

Morosan, D. E., Kilpua, E. K. J., Carley, E. P., \& Monstein, C. 2019b, A\&A, 623, A63

Morosan, D. E., Palmerio, E., Lynch, B. J., \& Kilpua, E. K. J. 2020a, A\&A, 633, A141

Morosan, D. E., Palmerio, E., Pomoell, J., et al. 2020b, A\&A, 635, A62

Nelson, G. J., \& Melrose, D. B. 1985, in Solar Radiophysics: Studies of Emission from the Sun at Metre Wavelengths (A87-13851 03-92), eds. D. J. McLean, \& N. R. Labrum (Cambridge and New York: Cambridge University Press), 333

Newkirk, G. J. 1961, ApJ, 133, 983

Ogilvie, K. W., \& Desch, M. D. 1997, Adv. Space Res., 20, 559

Owens, M. J. 2016, ApJ, 818, 197

Palmerio, E., Scolini, C., Barnes, D., et al. 2019, ApJ, 878, 37

Pesnell, W. D., Thompson, B. J., \& Chamberlin, P. C. 2012, Sol. Phys., 275, 3

Pomoell, J., Vainio, R., \& Kissmann, R. 2008, Sol. Phys., 253, 249

Reiner, M. J., Kaiser, M. L., \& Bougeret, J. L. 2007, ApJ, 663, 1369

Richardson, I. G., Lawrence, G. R., Haggerty, D. K., Kucera, T. A., \& Szabo, A 2003, Geophys. Res. Lett., 30, 8014

Saito, K., Poland, A. I., \& Munro, R. H. 1977, Sol. Phys., 55, 121

Sandroos, A., \& Vainio, R. 2006, A\&A, 455, 685

Santandrea, S., Gantois, K., Strauch, K., et al. 2013, Sol. Phys., 286, 5

Schrijver, C. J., \& De Rosa, M. L. 2003, Sol. Phys., 212, 165

Seaton, D. B., Berghmans, D., Nicula, B., et al. 2013, Sol. Phys., 286, 43

Stewart, R. T., Howard, R. A., Hansen, F., Gergely, T., \& Kundu, M. 1974, Sol. Phys., 36, 219

Stewart, R. T., Duncan, R. A., Suzuki, S., \& Nelson, G. J. 1978, Proc. Astron. Soc. Aust., 3, 247

Thernisien, A. F. R., Howard, R. A., \& Vourlidas, A. 2006, ApJ, 652, 763

Thernisien, A., Vourlidas, A., \& Howard, R. A. 2009, Sol. Phys., 256, 111

Török, T., Downs, C., Linker, J. A., et al. 2018, ApJ, 856, 75

van de Hulst, H. C. 1950, ApJ, 112,

Vasanth, V., Chen, Y., Lv, M., et al. 2019, ApJ, 870, 30

Vourlidas, A., Wu, S. T., Wang, A. H., Subramanian, P., \& Howard, R. A. 2003 , ApJ, 598, 1392

Vourlidas, A., Lynch, B. J., Howard, R. A., \& Li, Y. 2013, Sol. Phys., 284, 179

Vršnak, B., Aurass, H., Magdalenić, J., \& Gopalswamy, N. 2001, A\&A, 377, 321

Vršnak, B., Magdalenić, J., Aurass, H., \& Mann, G. 2002, A\&A, 396, 673

Vršnak, B., Klein, K.-L., Warmuth, A., Otruba, W., \& Skender, M. 2003, Sol. Phys., 214, 325

Wang, T., \& Davila, J. M. 2014, Sol. Phys., 289, 3723

Wang, Y. M., \& Hess, P. 2018, ApJ, 853, 103

Wang, Y. M., \& Sheeley, N. R. J. 1992, ApJ, 392, 310

Zhao, X., Liu, Y. D., Hu, H., \& Wang, R. 2019, ApJ, 882, 122

Zucca, P., Morosan, D. E., Rouillard, A. P., et al. 2018, A\&A, 615, A89 
D. E. Morosan et al.: Electron acceleration and radio emission resulting from two interacting coronal mass ejections

\section{Appendix A: Spectral properties of the moving radio bursts}

The moving radio bursts presented in this study correspond to fine structures in dynamic spectra observed by ORFEES. In this appendix, we show characteristic dynamic spectra for the three moving sources where we found fine-structured bursts in ORFEES corresponding to the NRH moving bursts intensity peaks. The ORFEES dynamic spectra, together with the NRH flux densities and ORFEES time series of the normalised intensity at 150 and $173 \mathrm{MHz}$, are shown in Figs. A.1-A.3 corresponding to Sources 1,2 , and 3, respectively. The white arrows in each figure point to the fine structures corresponding to the moving radio bursts.

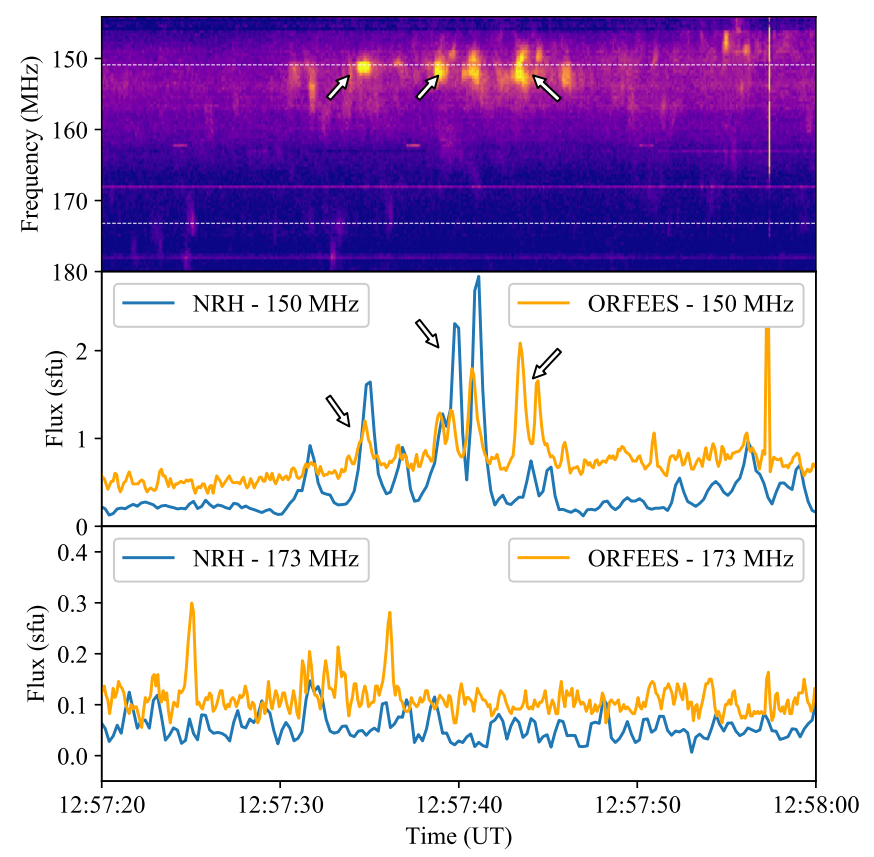

Fig. A.1. Spectral features corresponding to Source 1. (a) Zoomedin view of the NDA dynamic spectrum showing a 40-s time period starting from 12:57:20 UT. (b) Time series of the NRH flux density of Source 1 in sfu and ORFEES normalised intensity in arbitrary units at 150.9 MHz. (c) Time series of the NRH flux density of Source 1 in sfu and ORFEES normalised intensity in arbitrary units at $150.9 \mathrm{MHz}$.

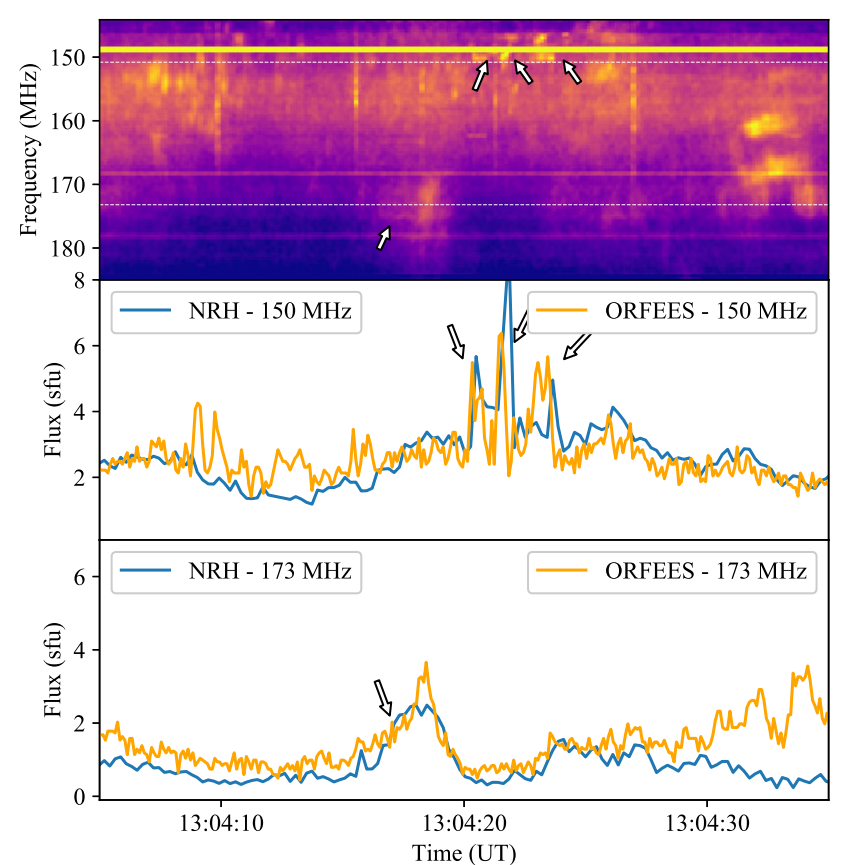

Fig. A.2. Spectral features corresponding to Source 2. (a) Zoomedin view of the NDA dynamic spectrum showing a 30-s time period starting from 13:04:05 UT. (b) Time series of the NRH flux density of Source 2 in sfu and ORFEES normalised intensity in arbitrary units at 150.9 MHz. (c) Time series of the NRH flux density of Source 2 in sfu and ORFEES normalised intensity in arbitrary units at $150.9 \mathrm{MHz}$.

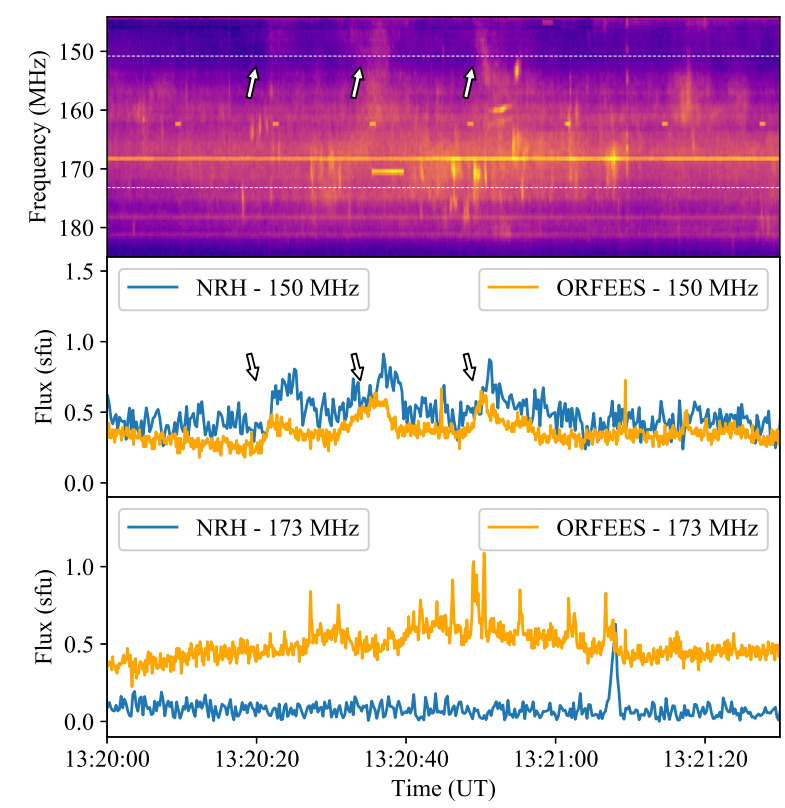

Fig. A.3. Spectral features corresponding to Source 3. (a) Zoomedin view of the NDA dynamic spectrum showing a 90-s time period starting from 13:20:00 UT. (b) Time series of the NRH flux density of Source 3 in sfu and ORFEES normalised intensity in arbitrary units at 150.9 MHz. (c) Time series of the NRH flux density of Source 3 in sfu and ORFEES normalised intensity in arbitrary units at $150.9 \mathrm{MHz}$. 